\title{
Development of a Method for Monitoring the Process of Heating the Lining of High-Temperature Units
}

\author{
Evgeniy V. Prikhodko* \\ Pavlodar State University named after S. Toraigyrov \\ Pavlodar, Kazakhstan
}

Received 04.03.2020, received in revised form 23.03.2020, accepted 09.04.2020

\begin{abstract}
This article proposes a new method for monitoring the thermal state of the lining of hightemperature units. Firstly, it is shown that the main methods for monitoring the thermal state of the lining are limited to obtaining data to prevent an emergency situation - leakage of the working medium through the lining and does not allow to obtain data on the temperature distribution over the cross section of the lining, for monitoring nonstationary thermal processes. Secondly, a method for determining the thermal state of the lining of a thermal unit is proposed, which allows one to obtain data on the temperature fields of the lining in the process of nonstationary heat conduction and use these values to control the process. Thirdly, to test the developed method, a physical model of the process of heating the lining of a high-temperature unit was created. Fourth, testing the physical model of the heating process showed that the developed method can be applied in practice, since its error does not exceed $10 \%$.
\end{abstract}

Keywords: lining, heating of high-temperature units, temperature control, four-point explicit difference scheme.

Citation: Prikhodko E.V. Development of a method for monitoring the process of heating the lining of high-temperature units, J. Sib. Fed. Univ. Eng. \& Technol., 2020, 13(4), 462-472. DOI: 10.17516/1999-494X-0233

(C) Siberian Federal University. All rights reserved

This work is licensed under a Creative Commons Attribution-Non Commercial 4.0 International License (CC BY-NC 4.0).

* Corresponding author E-mail address: john1380@mail.ru 


\title{
Разработка способа контроля \\ процесса разогрева футеровок \\ высокотемпературных агрегатов
}

\author{
Е.В. Приходько \\ Павлодарский государственный университет \\ им. С. Торайгырова \\ Казахстан, Павлодар
}

\begin{abstract}
Аннотация. В статье предложен новый метод для контроля теплового состояния футеровок высокотемпературных агрегатов. Во-первых, показано, что основные способы по контролю теплового состояния футеровок ограничиваются получением данных для недопущения аварийной ситуации - протечки рабочей среды через футеровку - и не позволяют получать данные о распределении температур по сечению футеровки, для контроля нестационарных тепловых процессов. Во-вторых, предложен способ определения теплового состояния футеровки теплового агрегата, который позволяет получать данные о температурных полях футеровки в процессе нестационарной теплопроводности и использовать эти величины для управления технологическим процессом. В-третьих, для апробации разработанного способа была создана физическая модель процесса разогрева футеровки высокотемпературного агрегата. В-четвертых, апробация физической модели процесса разогрева показала, что разработанный способ может применяться на практике, так как его погрешность не превышает $10 \%$.
\end{abstract}

Ключевые слова: футеровка, разогрев высокотемпературных агрегатов, контроль температуры, четырехточечная явная разностная схема.

Цитирование: Приходько, Е.В. Разработка способа контроля процесса разогрева футеровок высокотемпературных агрегатов / Е.В. Приходько // Журн. Сиб. федер. ун-та. Техника и технологии, 2020. 13(4). C. 462-472. DOI: 10.17516/1999$494 \mathrm{X}-0233$

\section{Введение}

Контроль теплового состояния высокотемпературных агрегатов является актуальной задачей при их эксплуатации. Это объясняется не только важностью бесперебойной (безаварийной) работы агрегатов, но и серьезностью аварийных ситуаций, связанных с разрушением футеровки. Так, прогар футеровки приводит не только к значительным материальным затратам, но и к травмированию эксплуатационного персонала $[1,2]$.

В данном случае ситуация усугубляется действием целого ряда факторов на толщину, а точнее говоря, профиль футеровки. При этом достаточно сложно обобщенно утверждать о более или менее важных факторах, влияющих на процессы износа. Это и температурный фактор, и немаловажный фактор физико-химических превращений в рабочем слое огнеупоров под влиянием технологических и эксплуатационных факторов.

Во многих случаях контроль теплового состояния футеровки ограничивается получением данных для недопущения аварийной ситуации - протечки рабочей среды через футеровку - и не позволяет получать данные о распределении температур по сечению футеровки для контроля нестационарных тепловых процессов. Информация о распределении температур по сечению футеровки важна для решения следующих задач:

$$
-463-
$$


- определения термонапряженного состояния футеровки в процессе эксплуатации (нестационарное и стационарное состояния);

- ведение нестационарных процессов (разогрева, охлаждения и др.) в соответствии с разработанными графиками;

- определение остаточной толщины слоя футеровки;

- прогнозирование остаточного ресурса футеровки и определение вероятности аварийной ситуации, связанной с нарушением герметичности футеровки;

- ряда других задач.

Современные способы контроля за тепловым состоянием либо имеют ограниченное применение только для узкого круга высокотемпературных агрегатов (например, индукционных печей), либо контролируют температуру только в одной точке. Отсутствие данных о распределении температур по сечению, особенно при нестационарных режимах, не позволяет решать часть вышеупомянутых задач.

Если говорить о контроле состояния футеровки в целом, то в первую очередь необходимо рассмотреть вопрос о проводимых обследованиях высокотемпературных агрегатов. Обследование состояния футеровки является неотъемлемой частью и первым этапом после остановки агрегата. При этом фиксируют следующие моменты:

- общее состояние футеровки - наличие расслоений, сколов, выпадения элементов футеровки;

- остаточная толщина футеровки и ее равномерность по агрегату (или зонам);

- наличие защитного слоя на поверхности (гарнисажа);

- состояние температурных швов и их заполнение.

Осмотр футеровки печи регламентируется нормативными документами, например [3].

Указанное мероприятие, как правило, носит характер фиксации уже существующих проблем и дает достаточно субъективные данные для оценки остаточного ресурса футеровки при дальнейшей работе, так как в этом случае невозможно предусмотреть все факторы (как внутренние, так и внешние), влияющие на стойкость футеровки. Таким образом, рассмотренный метод является первичным и предшествует всем остальным методам дефектоскопии. При этом для прогнозирования тепловой работы футерованных объектов данный вид имеет ограниченное применение, поскольку если состояние у футеровки характеризуется наличием визуальных трещин, то ряд агрегатов необходимо выводить в ремонт.

На некоторых установках, например дуговых сталеплавильных печах, тепловой контроль ограничивается установкой нескольких термопар (в зависимости от мощности печи) в футеровку. Данные термопары служат сигнализаторами разрушения футеровки более критического уровня и выполняют основную задачу - сигнал для слива металла из печи до момента проплавления его кожуха и выхода металла из рабочего пространства печи.

Более планомерный контроль предлагают вести авторы [4], где контроль разгара огнеупорной футеровки осуществляется с помощью датчиков, установленных в самой футеровке (электрический метод контроля). Работа способа основана на принципе нарушения или восстановления электрической цепи датчиков глубины по мере износа огнеупора либо на измерении температуры термопарами (при использовании термопар в качестве датчиков - термопарный метод). 
Датчики установлены непосредственно в огнеупорном кирпиче, в котором для датчиков выфрезерованы пазы, что является значительным недостатком этого метода. При использовании таких датчиков в высокотемпературных установках, работающих с расплавами металлов, возможно расплавление датчика и пролив металла через футеровку. То есть по этому критерию есть ограничение применения технологии - использование ее в агрегатах с расплавами относительно невысоких температур либо с газообразными средами (своды печей, стены над уровнем металла). Вторым недостатком способа считают сложность при их использовании в агрегатах с неравномерным износом. В таких случаях решением проблемы служит установка большого количества датчиков, что не всегда экономически оправдано.

Также частным решением указанных задач является исследование [5], где разработанная система контроля состояния футеровки горна позволяет визуализировать, диагностировать, контролировать тепловое состояние футеровки доменной печи с использованием математической модели 2D-3D. Результаты внедрения на доменных печах Китая дали положительный результат. Среди недостатков предлагаемой системы можно выделить то, что система разработана только для доменных печей и имеет значительное число датчиков температуры (до 700).

Немецкой компанией Saveway GmbH\&Co.KG разработана система, где на основе нелинейной зависимости электрического сопротивления огнеупоров от температуры производится визуализация остаточной толщины футеровки индукционной тигельной печи [6]. По мере работы, с уменьшением толщины футеровки расплав перемещается в сторону датчиков (электродных пластин), расположенных в толще футеровки. Вследствие этого увеличивается температура внутренней поверхности футеровки и изменяется ее удельное электрическое сопротивление.

Разработанная система предполагает визуализацию на программном обеспечении с сигнализацией критических состояний: «Предупреждение», «Критический износ». Предлагаемая система позволяет судить только лишь об износе футеровки, а не о ее тепловом состоянии. Но даже при использовании разработанной системы производитель заявляет о необходимости визуального контроля состояния тигля.

В последнее время все более широко применяют метод тепловизионного контроля состояния футеровки высокотемпературных агрегатов. Метод апробирован достаточно хорошо и на ряде агрегатов дает возможность получать достаточно точные данные о температурных полях поверхностей высокотемпературных агрегатов. Так, при использовании тепловизионного контроля для сталеразливочных ковшей авторы [7] говорят о возможности проведения динамических измерений температурных полей, что позволяет проводить тепловые расчеты конвективного и радиационного теплообмена. Итогом применения разработанной системы станет оценка прогаров футеровки и их прогнозирование, а также оценка теплосодержания расплава.

К недостаткам методики тепловизионного контроля можно отнести значительные затраты на разработанные установки тепловизионного контроля. Так, в работе [8] предлагается установить пять тепловизионных модулей для просмотра поверхности сталеразливочных ковшей и один пирометрический блок для оценки теплосодержания.

К недостаткам можно также отнести значительное количество факторов, влияющих на точность измерений:

- технологические процессы в самом агрегате (неравномерное распределение технологического материала);

$$
-465-
$$


- изменение степени черноты поверхности агрегата;

- возможное наличие различных сред между тепловизором и объектом исследования пар, газы, пыль и др.;

- воздействие внешних факторов - температура окружающей среды, солнечное излучение и др.

\section{Методика проведения исследований}

Нами разработан способ определения теплового состояния футеровки теплового агрегата, который позволяет получать данные о температурных полях футеровки в процессе нестационарной теплопроводности и использовать эти величины для управления технологическим процессом [9].

На рис. 1 изображена схема расположения датчиков в футеровке. Футеровка теплового агрегата представляет собой стенку, состоящую из кожуха 1 и слоя теплоизоляционного (или огнеупорного) материала 2. В футеровке размещены датчик температуры 3 на расстоянии $\mathrm{L}_{1}$ от ее внутренней поверхности и датчик температуры 4 на расстоянии $\mathrm{L}_{2}$ от ее внутренней поверхности. Внутри теплового агрегата находится греющая среда с температурой $\mathrm{T}_{0}$. Снаружи температура окружающей среды составляет значение $\mathrm{T}_{\mathrm{oc}}$.

В процессе кладки футеровки тепловых агрегатов в футеровку устанавливают датчики температуры на заданные расстояния от ее внутренней поверхности. Количество датчиков и расстояние от внутренней поверхности выбирают исходя из эксплуатационных ограничений, связанных с возможностью аварийных ситуаций (нарушением герметичности, протечкой металла и др.).

Для функционирования разработанной методики необходимы следующие исходные данные:

- характеристики материала (справочные данные): коэффициент температуропроводности, $\mathrm{M}^{2} / \mathrm{c}$; коэффициент теплопроводности, Вт/(м. $\left.{ }^{\circ} \mathrm{C}\right)$;

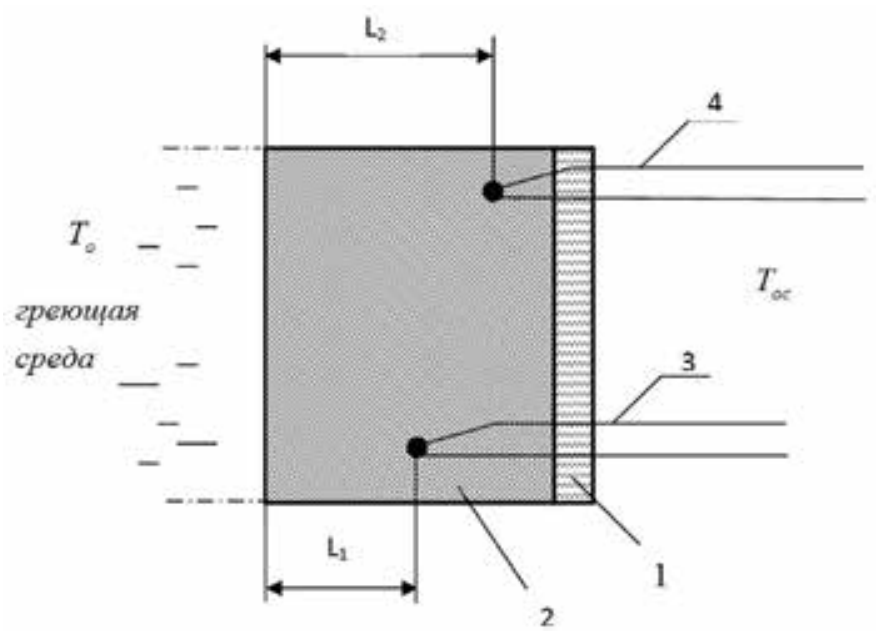

Рис. 1. Схема расположения датчиков температуры в футеровке

Fig. 1. Sceme of temperature sensors in the lining

$$
-466-
$$


- характеристики условий нагрева: коэффициент теплоотдачи, Вт/(м $\left.{ }^{2 . \circ} \mathrm{C}\right)$; температура греющей среды, ${ }^{\circ} \mathrm{C}$; время с начала разогрева, с.

Чем больше датчиков температуры располагают по толщине футеровки, тем меньше погрешность при определении температурного поля. Минимальное количество датчиков температуры обуславливается эксплуатационными ограничениями, связанными с возможностью аварийных ситуаций.

Для дальнейших расчетов принимают, что температура по сечению футеровки перед процессом нагрева одинакова во всех точках и равна температуре окружающей среды.

Далее задается первоначальная температура греющей среды $\mathrm{T}_{0}$. Первоначальное значение этой температуры принимают минимальным из возможных (при данных условиях нагрева). Затем производят расчет значений температур по сечению футеровки теплового агрегата для момента времени $\tau_{1}$ по любой разностной схеме.

Для явной четырехточечной схемы методика расчета будет выглядеть следующим образом. Задаются значением шага по времени $\Delta \tau$ и шага по координате $\Delta y$.

Вычисляют коэффициенты $b$ и $f$ по формулам

$$
b=\frac{\alpha \Delta y}{\lambda}, f=\frac{a \Delta \tau}{\Delta y^{2}},
$$

где $a$ - коэффициент температуропроводности, $\mathrm{M}^{2} / \mathrm{c} ; \alpha-$ коэффициент теплоотдачи, Вт/(м $\left.{ }^{2 . \circ} \mathrm{C}\right)$; $\Delta y$ - шаг по координате, м; $\Delta \tau$ - шаг по времени, м; $\lambda$ - коэффициент теплопроводности, $\mathrm{BT} /\left(\mathrm{M}^{\circ} \mathrm{C}\right)$.

Коэффициенты температуропроводности, теплоотдачи и теплопроводности являются справочными данными, которые находят по таблицам и диаграммам в зависимости от свойств материала и условий нагрева. Затем проверяют явную четырехточечную схему по условию сходимости:

$$
f \leq 1 /[2 \cdot(1+b)] .
$$

Если условие не выполняется, изменяют шаг по координате или времени, а если условие выполняется, то рассчитывают температуры по сечению в процессе разогрева от момента времени $\tau_{0}$ до момента времени $\tau_{1}$ с шагом по времени $\Delta \tau: k ; k+1 ; k+2$.

При этом температуру точки, соприкасающейся с греющей средой, определяют по формуле

$$
T_{n+1}^{k+1}=2 f T_{n}^{k}+[1-2 f(1+b)] T_{n+1}^{k}+2 f b T_{0}
$$

А температуру остальных точек по сечению в момент времени $k+1$ вычисляют по формуле

$$
T_{i}^{k+1}=f T_{i-1}^{k}+(1-2 f) T_{i}^{k}+f T_{i+1}^{k} .
$$

Если в рассматриваемый момент времени рассчитываемая температура в точке на расстоянии $\mathrm{L}_{1}$ от внутренней поверхности футеровки окажется равной $\mathrm{T}_{3}$ и температура в точке на расстоянии $\mathrm{L}_{2}$ от внутренней поверхности футеровки окажется равной $\mathrm{T}_{4}$, то расчет прекращают. При этом полученное распределение температур по сечению футеровки будет искомым.

$$
-467-
$$


Если же это условие не будет выполнено, задают другую температуру $T_{o}$ и вновь повторяют расчет.

\section{Лабораторные исследования}

Для проведения лабораторных измерений была поставлена задача создать физическую модель, которая отражала бы в необходимой мере процесс разогрева футеровки высокотемпературного агрегата. При этом были выделены следующие параметры, значения которых необходимо поддерживать в заданных пределах:

1. Передача теплоты в футеровке теплопроводностью. В данном отношении при исследованиях необходимо было добиться условия протекания в физической модели нестационарного процесса теплопроводности. Процесс стационарной передачи тепла не является определяющим в рассматриваемых процессах сушки и разогрева, поэтому при наступлении момента постоянства температур в сечении футеровки процесс измерения необходимо было прекратить.

2. Тепловой поток. Процесс сушки и разогрева футеровки с использованием топливных горелок предполагает постоянное (по крайней мере, в течение определенного времени) значение теплового потока. Для исследований предполагалось, что рассматривается временной участок, на котором значение теплового потока (температуры греющей среды) постоянно.

3. Свойства исследуемого материала. Для получения адекватных значений при измерениях необходимо было использовать материал, теплофизические и термомеханические параметры которого оставались бы без изменения (или изменялись в незначительной степени) в рассматриваемом диапазоне температур.

4. Место расположения датчиков температуры. Точки расположения датчиков должны находиться внутри слоя исследуемого материала и согласовываться с разработанной программой (математический эксперимент) исходя из «шага по координате».

5. Температура греющей среды. Для проведения измерений параметр «температура» не являлся определяющим, так как рассматривался нестационарный процесс разогрева высокотемпературных агрегатов, при котором идет повышение температуры футеровки с начального значения - температуры в цехе - до максимального значения, устанавливаемого технологией эксплуатации данного агрегата. Таким образом, диапазон исследуемых температур мог находиться в широких пределах.

6. Температура внешней среды. При проведении измерений создаваемый тепловой поток не должен оказывать сколько-нибудь значительное воздействие на температуру воздуха в помещении.

Таким образом, с учетом вышеприведенных условий была создана физическая модель процесса (исследовательская установка) (рис. 2). В качестве источника теплоты была использована муфельная печь 1. Исследуемым материалом служили два слоя (общей толщиной 90 мм) матов из базальтового волокна. Для измерения температур применяли три термопары. Первая термопара (3) проходила сквозь два слоя базальта для измерения температуры внутри печи. Вторая термопара (4) устанавливалась внутри теплоизоляционного слоя на определенном расстоянии от камеры печи. Третья термопара (5) располагалась на заданном расстоянии от второй термопары. 


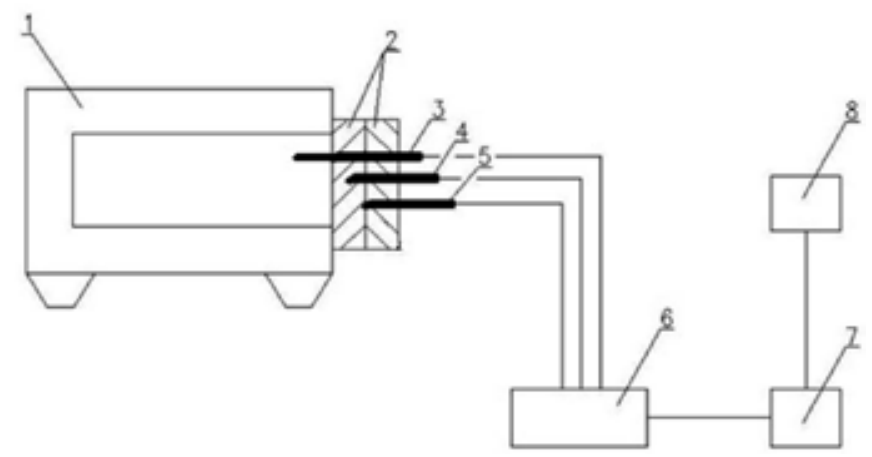

Рис. 2. Исследовательская установка

Fig. 2. Research facility

Для регистрации сигналов термопар использовали универсальный измеритель-регулятор ТРМ-138 (6) и адаптер интерфейса (7) АC-3. Все устройства подключали к компьютеру (8), и при помощи программы «SCADA. OWEN PROCESS MANAGER» обрабатывалась информация об изменениях.

Методика заключалась в следующем. Разогревали муфельную печь до температуры исследования. При этом в дальнейшем температуру печи поддерживали на постоянном уровне, что создавало постоянный тепловой поток. После выхода температуры внутри печи на стационарное состояние производили открытие дверцы печи и вместо дверцы помещали исследуемый материал с размещенными в нем термопарами. Тем самым соблюдалось условие постоянства теплового потока греющей среды.

На рис. 3 приведен пример отображения измеряемых параметров в программе OWEN PROCESS MANAGER.

Для выбранных данных необходимо проверить условие сходимости по формуле (2) :

$$
\begin{aligned}
& f \leq 1 /[2 \cdot(1+b)], \\
& 0,0024 \leq 0,011 .
\end{aligned}
$$

Таким образом, схема является устойчивой при указанных исходных данных.

Погрешность аппроксимации явной разностной схемы (сходимость) равна:

$$
v=O\left(\Delta y^{2}+\Delta \tau\right)
$$

т.е. для определения погрешности расчета вычисляем распределение температур в слое исследуемого материала при увеличении шага по времени в 4 и координате в 2 раза. Соответственно, шаг по координате $\Delta y$ составит $0,01125 \mathrm{M}$, а шаг по времени $\Delta \tau=0,25 \mathrm{c}$.

При этом имеющееся условие сходимости будет соблюдаться:

$$
\begin{aligned}
& f \leq 1 /[2 \cdot(1+b)], \\
& 0,0024 \leq 0,026 .
\end{aligned}
$$

В рассматриваемом примере необходимо выбрать узел, для которого разность между решениями, найденными в первом и втором вариантах, имеет наибольшее значение, и применить

$$
-469-
$$




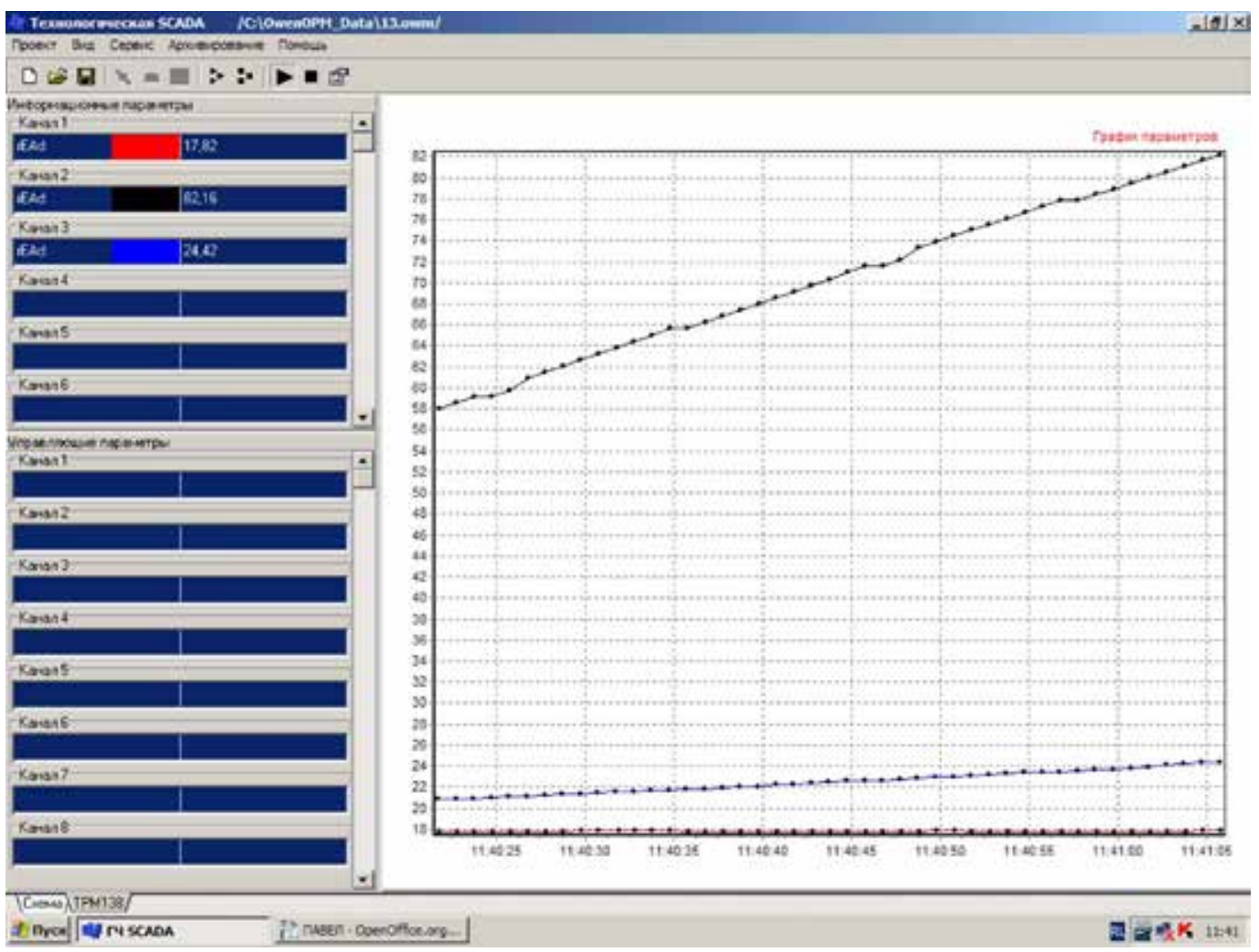

Рис. 3. Отображение измеряемых параметров в программе OWEN PROCESS

Fig. 3. Display of measured parameters in OWEN PROCESS

для него метод Рунге. После произведенных расчетов получаем, что разность температур для точки, расположенной на расстоянии 0,0225 м от границы внешней стенки, будет достигать максимального значения в момент времени $150 \mathrm{c}$.

Вычисляем погрешность определения температур футеровки в этой точке, ${ }^{\circ} \mathrm{C}$ :

$$
\varepsilon \approx \frac{4}{3} \cdot(31,4-30,27) \approx 1,51 \text {. }
$$

Для точки, расположенной на расстоянии 0,045 м от границы внешней стенки, разность температур будет достигать максимального значения в момент времени $130 \mathrm{c}$.

Вычисляем погрешность определения температур футеровки в этой точке, ${ }^{\circ} \mathrm{C}$ :

$$
\varepsilon \approx \frac{4}{3} \cdot(101,49-99,45) \approx 2,7 .
$$

Кроме определения погрешности численного метода определения температур по сечению, можно также подсчитать максимальную разность между значениями температуры, вычисленными по программе и измеренными на физической модели.

Разность температур для точки, расположенной на расстоянии 0,0225 м от границы внешней стенки, будет достигать максимального значения в момент времени $260 \mathrm{c},{ }^{\circ} \mathrm{C}$ :

$$
\Delta t=53-48,7=4,3 .
$$


Разность температур для точки, расположенной на расстоянии 0,045 м от границы внешней стенки, будет достигать максимального значения в момент времени $280 \mathrm{c},{ }^{\circ} \mathrm{C}$ :

$$
\Delta t=160,72-152,6=8,12 .
$$

\section{Выводы}

Таким образом, разработан способ контроля процесса разогрева футеровок высокотемпературных агрегатов, который позволяет с достаточно высокой точностью (погрешность не более 10 \%) определять температуры по сечению футеровки в процессе разогрева.

Апробация физической модели процесса разогрева показала, что математическая модель может применяться на практике. Для решения одномерной задачи теплопроводности в качестве расчетной схемы используется четырехточечная явная разностная схема, имеющая относительно высокую точность и простоту. Разработанная схема может применяться для контроля разогрева разливочных ковшей, различного рода печей с ограничением по глубине установки датчиков температуры.

\section{Список литературы / References}

[1] Годовой отчет о деятельности Федеральной службы по экологическому, технологическому и атомному надзору в 2016 году. М., 2017, 397 с. [Annual report on the activities of the Federal Service for Ecological, Technological and Nuclear Supervision in 2016. М., 2017, 397 p. (in Russian)]

[2] В результате аварии на АЗФ в Павлодарской области пострадали двое рабочих [Электронный ресурс] - Режим доступа: https://tengrinews.kz/events/rezultate-avarii-azf-pavlodarskoyoblasti-postradali-dvoe-196862/ [As a result of the accident at the Aksu Ferroalloy Plant in Pavlodar region, two workers were injured - Access: https://tengrinews.kz/events/rezultate-avarii-azfpavlodarskoy-oblasti-postradali-dvoe-196862/ (in Russian)]

[3] Методика определения остаточного ресурса трубчатых печей нефтеперерабатывающих, нефтехимических и химических производств. ОАО «ВНИКТИнефтехимоборудование». Волгоград, 1998. 34 c. [Methodology for determining the residual life of tube furnaces in oil refining, petrochemical and chemical industries. OJSC "VNIKTIneftekhimoborudovanie", Volgograd, 1998, 34 p. (in Russian)]

[4] Харламов Д.А., Коберник О.П., Ансимов А.А. Методы контроля разгара огнеупорной футеровки в промышленных печах. Фундаментальные исследования. 2013, 6-4, 877-879. [Kharlamov D.A., Kobernik O.P., Ansimov A.A. Methods of control of the peak refractory lining of industrial furnaces. Fundamental research. 2013, 6-4, 877-879. (in Russian)]

[5] Дмитриев А.Н., Золотых М.О., Чэнь К., Витькина Г.Ю. Сравнительный технологический анализ систем контроля разгара огнеупорной футеровки горна доменной печи. Черная металлургия. Бюллетень научно-технической и экономической информачии. 2018, (8), 8291. [Dmitriev A.N., Zolotykh M.O., Chen' K., Vit'kina G.Y. Comparative technological analysis of control systems of blast furnace hearth lining deterioration. Ferrous Metallurgy. Bulletin of Scientific, Technical and Economic Information. 2018, (8), 82-91 (in Russian)]

[6] Система SAVEWAY. Непрерывное измерение остаточной толщины футеровки. [Электронный ресурс] - Режим доступа: https://www.saveway-germany.de/ru/2284-2/saveway/

$$
-471-
$$


[SAVEWAY ${ }^{\circledR}$ system. Continuous measurement of remaining lining thickness - Access: https://www. saveway-germany.de/ru/2284-2/saveway/ (in Russian)]

[7] Рыбаков А.Г., Коробейников С.М., Цуприк Л.С. Применение теплового неразрушающего контроля футеровки миксеров для перевозки жидкого чугуна с помощью стационарных тепловизионных камер. Современные научные исследования и инновации. 2015, 6, 1 [Rybakov A.G., Korobeynikov S.M., Tsuprik L.S. Thermal nondestructive testing application for liquid iron transportation mixer lining control via stationary thermal cameras. Modern scientific researches and innovations. 2015, 6, 1 [Electronic journal] (in Russian)]

[8] Системы контроля зон возможного прогара футеровки сталеналивочных ковшей. [Электронный ресурс] - Режим доступа: http://ntc-pribor.ru/project/38-sistemy-kontrolya-zonvozmozhnogo-progara-futerovki-stalenalivochnyh-kovshey.html [Control systems for zones of possible burnout of the lining of steel ladles - Access: http://ntc-pribor.ru/project/38-sistemy-kontrolya-zonvozmozhnogo-progara-futerovki-stalenalivochnyh-kovshey.html (in Russian)]

[9] Инновационный патент № 26932 Республика Казахстан, МКИ G 01 К 13/00. Способ определения теплового состояния футеровки теплового агрегата. Никифоров А.С., Приходько Е.В., Кинжибекова А.К., Кучер Е.О., Никонов Г.Н., заявл. 06.08.12, опубл. 15.05.2013, бюл. № 5 [Innovative patent №. 26932 Republic of Kazakhstan IPC G 01 K 13/00. Method for determining the thermal state of the lining of a thermal unit. Nikiforov A.S., Prikhodko E.V., Kinzhibekova A.K., Kucher E.O., Nikonov G.N. appl.. 06.08.12, publ. 15.05.2013, Bull. Number 5 (in Russian)] 